\title{
NONNEGATIVE MATRICES EACH OF WHOSE POSITIVE DIAGONALS HAS THE SAME SUM
}

\author{
MARK BLONDEAU HEDRICK ${ }^{1}$
}

\begin{abstract}
The author shows that if $A$ is a fully indecomposable nonnegative matrix each of whose positive diagonals has sum $M$ and when $a_{i j}=0$, the sum of each positive diagonal in the submatrix of $A$ obtained by deleting the $i$ th row and $j$ th column is less than $M$, then there is a unique positive matrix $B$ such that its rank is at most two, each of its diagonals has sum $M$, and $a_{i j}=b_{i j}$ when $a_{i j}>0$. The author then compares his results to those obtained by Sinkhorn and Knopp who carried out a similar analysis for positive diagonal products.
\end{abstract}

Introduction and definitions. In [3], Sinkhorn and Knopp showed that if $A$ is a fully indecomposable nonnegative $n \times n$ matrix each of whose positive diagonal products is equal, then there is a unique positive matrix $B$ such that $b_{i j}=a_{i j}$ whenever $a_{i j}>0$ and $B$ is of rank one. Using the above result and another result proved in [3], they showed that no two doubly stochastic matrices have corresponding diagonal products proportional. In [1], Hartfiel showed that no two doubly stochastic matrices have corresponding diagonal sums equal. Thus the author became interested in examining just how well the diagonal sums of a matrix characterize it.

The main result is the following. Suppose $A$ is a fully indecomposable nonnegative $n \times n$ martix each of whose positive diagonals has sum $M$ and whenever $a_{i j}=0$, each positive diagonal in $A(i \mid j)$ has sum less than $M$. Then there exists a unique positive matrix $B$ such that the sum of each diagonal is $M, b_{i j}=a_{i j}$ whenever $a_{i j}>0$, and its rank is at most two.

One of the main tools which the author uses to obtain the above result is an immediate corollary of the Frobenius-König Theorem. A proof of the Frobenius-König Theorem can be found in [2, pp. 97-98].

FrobeniUs-KöNig Theorem. Every diagonal of an $n \times n$ matrix $A$ contains a zero element if and only if $A$ has an $s \times t$ zero submatrix with $s+t=n+1$.

COROLlaRY. If $A$ is a fully indecomposable nonnegative $n \times n$ matrix, then for each $1 \leqslant i, j \leqslant n, A(i \mid j)$ contains a positive diagonal.

Presented to the Society, October 31, 1970 under the title Nonnegative matrices each of whose positive diagonals has the same sum; received by the editors July 17, 1970 and, in revised form, December 28, 1974.

AMS (MOS) subject classifications (1970). Primary 05B20, 15A15.

Key words and phrases. Fully indecomposable, doubly stochastic pattern, elementary symmetric function of a diagonai.

${ }^{1}$ This author was partially supported by NDEA Title IV Grant 67-11015. 
Let us now define the notions which we shall need. A diagonal of a square matrix is a collection of entries from the matrix, one from each row and one from each column. If $\sigma$ is a permutation of $\{1, \ldots, n\}$, then the diagonal associated with $\sigma$ is $a_{1 \sigma(1)}, \ldots, a_{n \sigma(n)}$. Every diagonal corresponds to a permutation. Let $\sigma$ be a diagonal $a_{1 \sigma(1)}, \ldots, a_{n \sigma(n)}$ in the matrix $A$. Then we shall say that the diagonal $\sigma$ is positive if $a_{i \sigma(i)}$ is positive for each $i$. By the sum of the diagonal $\sigma$, we mean $\sum_{k=1}^{n} a_{k \sigma(k)}$.

Let $\alpha$ and $\beta$ be sequences of integers chosen from $\{1, \ldots, n\}$ and let $A$ be an $n \times n$ matrix. Then $A[\alpha \mid \beta]$ is a submatrix of $A$ containing rows $\alpha$ and columns $\beta$ and $A(\alpha \mid \beta)$ is a submatrix of $A$ containing rows complementary to $\alpha$ and columns complementary to $\beta$, ordered increasingly. By $A^{T}$ we mean the matrix whose $(i, j)$ entry is the $(j, i)$ entry of $A$. We shall let $E_{i j}$ denote the square matrix which has the number one in the $(i, j)$ position and zeros elsewhere.

An $n \times n$ matrix $A$ is fully indecomposable if and only if there does not exist an $s \times t$ zero submatrix of $A$ such that $s+t=n$. Otherwise, $A$ is said to be partly decomposable. A square nonnegative matrix $A$ is said to be nearly decomposable if and only if $A$ is a fully indecomposable matrix such that for each positive $a_{i j}, A-a_{i j} E_{i j}$ is partly decomposable. By convention we shall let the $1 \times 1$ zero matrix be partly decomposable. Suppose $A$ and $B$ are $n \times n$ matrices such that $a_{i j}=0$ if and c.n.y if $b_{i j}=0$. Then $B$ is said to have the same zero pattern as $A$. A matrix $A$ is said to have doubly stochastic pattern if there is a doubly stochastic matrix which has the same zero pattern as $A$.

Results and consequences. Let us first prove the Corollary to the FrobeniusKönig Theorem. If $A(i \mid j)$, for some $i$ and $j$, did not contain a positive diagonal, then $A$ would be partly decomposable by the Frobenius-König Theorem.

THEOREM 1. Suppose $A$ is a nonnegative fully indecomposable $n \times n$ matrix each of whose positive diagonals has sum $M$ and whenever $a_{i j}=0$, each positive diagonal in $A(i \mid j)$ has sum less than $M$. Then there is a unique positive matrix $B$ such that its rank is at most two, each of its diagonals has sum $M$, and $b_{i j}=a_{i j}$ whenever $a_{i j}>0$.

Proof. Let $C$ be the $n \times n$ fully indecomposable matrix where $c_{i j}$ $=\exp \left(a_{i j}\right)$ for $a_{i j}>0$ and $c_{i j}=0$ for $a_{i j}=0$. Thus, by the theorem in [3] (quoted in the Introduction), there is a unique positive matrix $C^{\prime}$ each of whose diagonal products equals $\exp (M)$ and $c_{i j}^{\prime}=c_{i j}$ whenever $c_{i j}>0$. Thus every diagonal in the $n \times n$ matrix $B=\left(b_{i j}\right)=\left(\ln c_{i j}^{\prime}\right)$ has sum $M$. Observe that $b_{i j}=a_{i j}$ for $a_{i j}>0$. Consider $b_{i j}=\ln \left(c_{i j}^{\prime}\right)$ for $a_{i j}=0$. By the Corollary, there is a positive diagonal $a_{1 \sigma(1)}, \ldots, a_{n \sigma(n)}$ in $A(i \mid j)$. Hence

$$
\begin{aligned}
c_{i j}^{\prime}\left(\Pi_{k=1, k \neq i}^{n} c_{k \sigma(k)}^{\prime}\right) & =\exp (M), \\
c_{i j}^{\prime}\left(\exp \left(\sum_{k=1, k \neq i}^{n} a_{k \sigma(k)}\right)\right) & =\exp (M), \text { and } \\
c_{i j}^{\prime} & =\exp \left(M-\sum_{k=1, k \neq i}^{n} a_{k \sigma(k)}\right) .
\end{aligned}
$$


Since $M-\sum_{k=1, k \neq i}^{n} a_{k \sigma(k)}>0, b_{i j}=\ln \left(c_{i j}^{\prime}\right)>0$. Thus $B$ is positive. Since the exp function is $1-1$, the uniqueness of $B$ follows from the uniqueness of $C^{\prime}$.

Let $D=\left(d_{i j}\right)$ be a $3 \times 3$ submatrix of $B$. Since all the diagonal sums of $B$ are equal, all the diagonal sums of every $2 \times 2$ submatrix of $B$ are equal. Thus $d_{i j}=d_{i-1, j}+\left(d_{i, j+1}-d_{i-1, j+1}\right)$ for $2 \leqslant i \leqslant 3$ and $1 \leqslant j \leqslant 2$. Perform the following operations in the order indicated: Subtract the 2nd row from the 3 rd row and then the 1 st row from the 2 nd row. Subtract the 2 nd column from the 1 st column and then the 3 rd column from the 2 nd column. The resulting matrix $D^{\prime}$ has $d_{i j}^{\prime}=0$ for $i \neq 1$ and $j \neq 3$. Hence $\operatorname{det} D^{\prime}=0$ which implies det $D=0$. Thus the rank of $B$ is at most two.

Corollary 1. Suppose $A$ is a fully indecomposable nonnegative $n \times n$ matrix each of whose positive diagonals has sum $M$ and whenever $a_{i j}=0$, the sum of each positive diagonal in $A(i \mid j)$ is less than $M$. Then if $C$ is a square submatrix of $A$, there is a number $N(C)$ such that each of the positive diagonals of $C$ has sum $N(C)$.

CoROllary 2. Suppose $A$ is an $n \times n$ nonnegative matrix which has doubly stochastic pattern. Assume that each of $A$ 's positive diagonals has sum $M$ and whenever $a_{i j}=0$, each positive diagonal in $A(i \mid j)$ has a sum which is less than $M$. Then if $C$ is a square submatrix of $A$, there is a number $N(C)$ such that each of the positive diagonals of $C$ has sum $N(C)$.

Proof. As pointed out in [3] and [4, p. 346], since $A$ has doubly stochastic pattern, there are permutation matrices $P$ and $Q$ such that $P A Q$ $=D_{1} \oplus \cdots \oplus D_{s}$ where each $D_{m}$ is fully indecomposable for $m=1, \ldots, s$. Assume that $A=D_{1} \oplus \cdots \oplus D_{s}$. Let us argue that each $D_{m}$ satisfies the hypothesis of Corollary 1 . Let $1 \leqslant k \leqslant s$ be fixed. Observe that the sum of each positive diagonal of $D_{k}$ is equal. Let $d_{k}$ and $e_{k}$ be two positive diagonals in $D_{k}$, and let $d_{m}$ be a positive diagonal in $D_{m}$ for $m=1, \ldots, s$ and $m \neq k$. We know that each of these diagonals exists by the Corollary to the Frobenius-König Theorem. Let $M_{m}$ be the sum of the diagonal $d_{m}$ for $m=1, \ldots, s$. By hypothesis, the sum of $d_{k}$ plus the sum of the $M_{m}$ 's for $m=1, \ldots, s$ and $m \neq k$ equals the sum of $e_{k}$ plus the sum of the $M_{m}$ 's for $m=1, \ldots, s$ and $m \neq k$. Thus the sum of $d_{k}$ equals the sum of $e_{k}$. Let $d_{p q}$ be a zero entry of $D_{k}$ which lies in the $(u, v)$ position of $A$. Let $d^{\prime}$ be a positive diagonal in $D_{k}(p \mid q)$ which we know to exist by the Corollary. Thus $d^{\prime} \cup$ $\left(\cup_{m=1, m \neq k} d_{m}\right)=t$ is a positive diagonal in $A(u \mid v)$. By hypothesis, the sum of $t$ is less tha.l $M$. Since the sum of $t$ is less than $M=\sum_{m=1}^{s} M_{m}$ and the sum of $t$ is equal to $\sum_{m=1, m \neq k}^{s} M_{m}$ plus the sum of $d^{\prime}$, it follows that the sum of $d^{\prime}$ is less than $M_{k}$.

Let $A[\alpha \mid \beta]$ be any square submatrix of $A$. In order for $A[\alpha \mid \beta]$ to have a positive diagonal, it must be of the form $D_{1}^{\prime} \oplus \cdots \oplus D_{r}^{\prime}$ where $r \leqslant s$ and each $D_{p}^{\prime}$ is a submatrix of some $D_{m}, 1 \leqslant p \leqslant m \leqslant s$. Thus by using Corollary 1 on each $D_{m}$, we are finished.

The following matrix satisfies the hypothesis of Theorem 1.

$$
A=\left[\begin{array}{lll}
2 & 0 & 2 \\
1 & 2 & 0 \\
0 & 2 & 1
\end{array}\right]
$$


The $B$ corresponding to $A$ by Theorem 1 is

$$
B=\left[\begin{array}{lll}
2 & 3 & 2 \\
1 & 2 & 1 \\
1 & 2 & 1
\end{array}\right]
$$

Notice that the positive matrix

$$
C=\left[\begin{array}{lll}
2 & 4 & 2 \\
1 & 2 & 1 \\
1 & 2 & 1
\end{array}\right]
$$

which corresponds to $A$ by the result in [3] (quoted in the first paragraph of this paper) is different from $B$. However, in case the two matrices are the same, the following theorem describes the form of the positive matrix.

Theorem 2. Suppose $A$ is a positive square matrix each of whose diagonal sums is equal and whose rank is one. Then either $A$ or $A^{T}$ has the form

$$
\left[\begin{array}{cccccc}
a_{1} & a_{1} & \ldots & a_{1} & a_{1} \\
a_{2} & a_{2} & \ldots & a_{2} & a_{2} \\
\cdots & \ldots & \ldots & \ldots & \ldots & . \\
a_{n} & a_{n} & \ldots & a_{n} & a_{n}
\end{array}\right]
$$

Proof. Suppose there is a row of $A$ in which all of the entries are the same. Then for any other row, the entries will be the same since the two diagonals in any $2 \times 2$ submatrix of $A$ have equal sums. Suppose that there is a row $k$ such that for some $p$ and $q, a_{k p} \neq a_{k q}$. Let $1 \leqslant m \leqslant n$ be fixed. Since $A$ is of rank one, there is a $u>0$ such that for all $j, a_{m j}=u a_{k j}$. Since the two diagonals of any $2 \times 2$ submatrix of $A$ have equal sums, $a_{k p}+u a_{k q}=a_{k q}+$ $u a_{k p}$. Then $a_{k p}-a_{k q}=u\left(a_{k p}-a_{k q}\right)$ which entails $u=1$. Thus all the rows are the same. Hence in any column of $A$, the entries of that column are all the same.

Recalling that the diagonal products of an $n \times n$ positive matrix are all equal if and only if it is of rank one, we see that Theorems 1 and 2 and the result in [3, p. 71] (quoted in the Introduction) are actually statements about the 1 st and $n$th elementary symmetric functions.

Unfortunately, Theorem 1 cannot be proven for an arbitrary elementary symmetric function. For instance, consider the fully indecomposable matrix

$$
A=\left[\begin{array}{cccc}
1 & 0 & 0 & 2 \\
2 & 1 & 0 & 0 \\
0 & 9 / 5 & 3 & 0 \\
0 & 0 & 1 & 2
\end{array}\right]
$$

Observe that the 2nd elementary symmetric function on each of $A$ 's positive diagonals is equal to 17. Observe also that for $a_{i j}=0,\left[17-E_{2}(d)\right] / E_{1}(d)>0$ where $d$ is the positive diagonal in $A(i \mid j)$ and $E_{1}(d)$ and $E_{2}(d)$ are the 1st and 2nd elementary symmetric functions applied to $d$, respectively. Using the positive diagonals in $A(1 \mid 2)$ and $A(3 \mid 4)$, we see that if there were a positive matrix corresponding to $A$, necessarily $b_{12}=1 / 7$ and $b_{34}=14 / 3$. Thus $b_{12}$, $a_{21}, b_{34}, a_{43}$ is a positive diagonal in $B$. However, $E_{2}(1 / 7,2,14 / 3,1)=17+$ $2 / 21$. Thus there cannot be a positive matrix corresponding to $A$ in the sense 
that $b_{i j}=a_{i j}$ for $a_{i j}>0$ and the 2 nd elementary symmetric function on each diagonal is equal.

Professor Richard Sinkhorn has shown that if $A$ is $3 \times 3$, then there always exists such a $B$.

If a $B$ exists for an $A$, then it is clearly unique by the Corollary to the Frobenius-König Theorem.

\section{REFERENCES}

1. Darald Hartfiel, An inequality concerning a matrix function (oral communication).

2. Marvin Marcus and Henryk Minc, $A$ survey of matrix theory and matrix inequalities, Allyn and Bacon, Boston, Mass., 1964. MR 29 \#112.

3. Richard Sinkhorn and Paul Knopp, Problems involving diagonal products in nonnegative matrices, Trans. Amer. Math. Soc. 136 (1969), 67-75. MR 38 \#2151.

4. __ Concerning nonnegative matrices and doubly stochastic matrices, Pacific J. Math. 21 (1967), 343-348. MR 35 \# 1617.

Current address: Hedrick Consultant Firm, 222 Kalmer, Pasadena, Texas 77502 